\title{
Numerical analysis of damage kinetics in aluminium under axi- symmetric loading
}

\author{
Olga Tyupanova ${ }^{1, *}$, Sergey Nadezhin ${ }^{1}$, Pavel Duday ${ }^{1}$, and Andrey Ivanovsky ${ }^{1}$ \\ ${ }^{1}$ RFNC-VNIIEF, 607190 Mira ave., Sarov, Russian Federation
}

\begin{abstract}
Based on the analysis of the results of two R-Damage experiments, this paper provides a variant of a relation to simulate the spall damage recollection under the effect of weak compression waves. This relation closes the kinetic fracture model, considering both growth and recollection of damage. Using this model makes it possible to simulate adequately (with good quantitative agreement) the sequence of the processes "shock-wave compression - spall fracture - convergence of spall layer - recollection of damaged medium", including the dynamics of the compacting wave arrival to the internal surface of the hollow cylindrical targets since it passed through the damaged media.
\end{abstract}

\section{Introduction}

Among the important and topical problems of the mechanics of the deformed solids are the processes of dynamic deformation, spall fracture and recollection. One of the tasks on this area is to develop and validate both physico-mathematical models and numerical simulation methods to describe these processes. Comparative analysis of the numerical and experimental results allows stating the correctness of a model.

As an example of such validation of a model, the peculiarities of the damage medium behavior under weak compression were analyzed in this work with the help of numerical simulation. Data of two tests " $R$ Damage-8\&9" [1] representing the completion stage of the experimental series " $R$-Damage-0-9" [2] realized jointly by the VNIIEF and LANL teams were used. The damage evolution and damaged matter recollection under axis-symmetric convergence were studied in the extruded aluminum Al 1060 while these experiments.

\section{Set-up of experiments}

In the R-Damage series the hollow cylindrical targets with varying inner and outer diameters were loaded by an impact of the cylindrical liner driven by electromagnetic field generated with the use of the explosive magnetic source of pulsed power (EPPS). The EPPS comprised of a helical explosive magnetic generator (HEMG), an explosive current opening switch (OS), an explosive current interrupter (CI) and a coaxial transmission line (TL) supplied the current from the explosive magnetic device to the liner unit is described in [3]. The set-up of the R-Damage tests is presented in [1-4] in detail.

The principle used in the experiments and consisting in the liners' drive due to magnetic field pressure occurring at the flow of the current pulse of required form provides the simplicity of variation of their motion regime and, respectively, of the targets loading regime. To realize the current pulses with required duration, the explosive current interrupter (CI) was used.

In the described $R$-Damage- $8 \& 9$ experiments the $\mathrm{CI}$ functioned with a certain time delay ensuring the required duration of the current pulse $\sim 35 \mu$ s in the liner load (see Fig.1). Such characteristics of the current pulse $I(t)$ allowed getting a long drive of the liner and impacting the targets twice. As a result, the regime with two successive compression waves was realized in the targets. At this, the reflection of the first wave off the inner (free) surface of the target produces the interaction of two opposite rarefaction waves and the initiation of the spall damage, while the second wave reaccelerates the fractured target and recollects the damage.

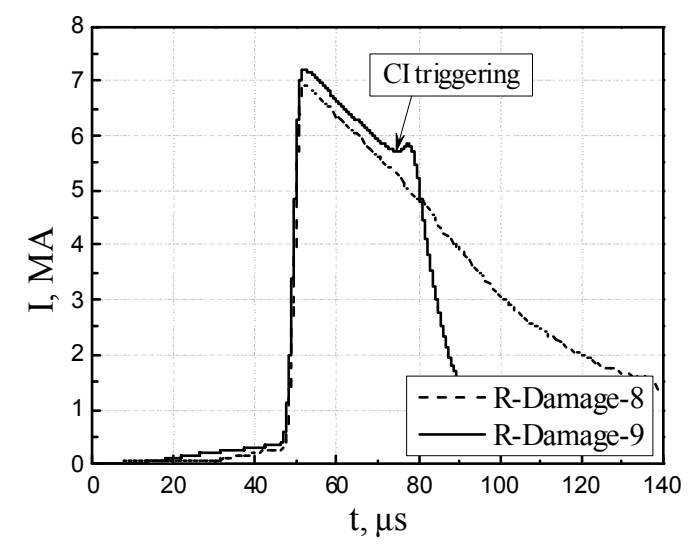

Fig. 1. Current vs time (Faraday probes, LANL).

Each experiment explored the response of two cylindrical targets of the same thickness $6 \mathrm{~mm}$. The targets were differed by the initial radii: target 1 (T1) $R_{\text {inner }}=50 \mathrm{~mm}$, target 2 (T2) $R_{\text {inner }}=49.5 \mathrm{~mm}$ (Fig. 2).

*Corresponding author: oatyupanova@vniief.ru 
The initial inner radius of the liner was $R_{\text {Liner }}=57 \mathrm{~mm}$ (with $3 \mathrm{~mm}$ thickness). To reduce axial release waves during the axis-symmetric motion, the hollow cylindrical targets were placed between two cylinders (momentum traps) with similar outer and inner radii (see Fig. 2). By using the specially designed protection system the liner units were recovered in each experiments that allowed to perform metallographic analysis of the post-shot state of the targets in order to reveal the degree of spall damage and its recollection extent (see the photo of the sectioned liner units in Fig. 3). To record the current parameters of the device in the experiments, the VNIIEF induction probes (IP) and the LANL Faraday probes (FP) were used. The liners' and the targets' inner surface velocity were recorded by PDV technique [5], with the velocity probes located in the central measuring unit (Fig.2).

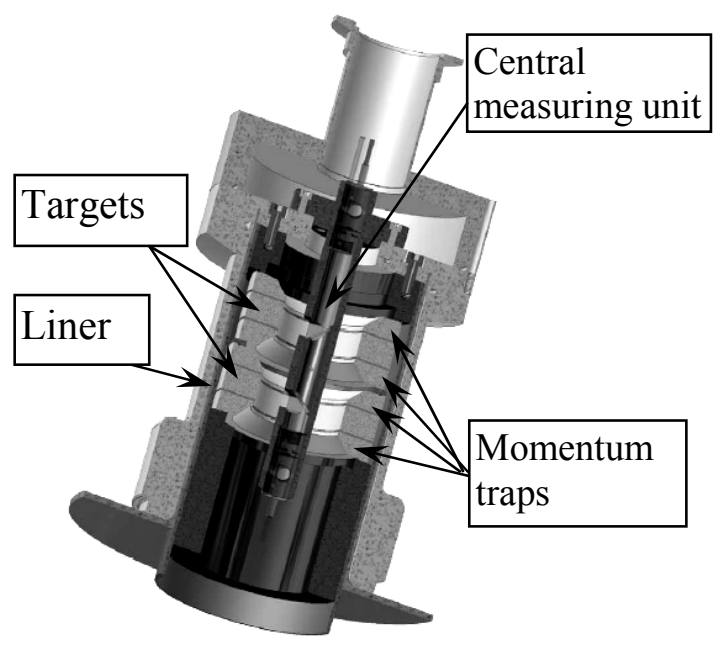

Fig. 2. Section of the liner unit.

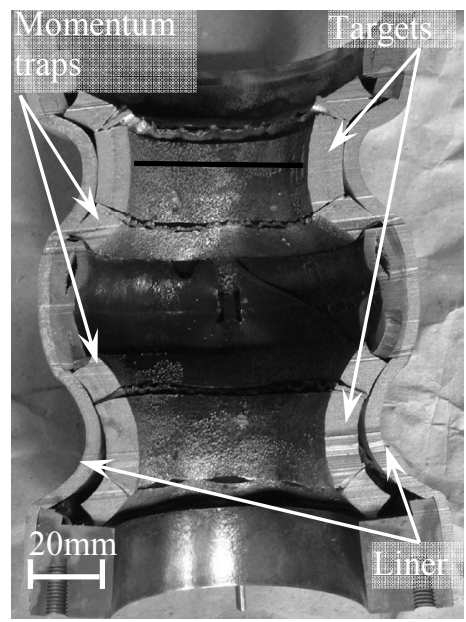

Fig. 3. Photo of the liner unit sectioned after the test $(R D-9)$.

Both the targets and the liner were made of the Alcan-manufactured high-purity aluminium Al-1060-O. Metallographic analysis of the pre-shot state of the material revealed a significant inhomogeneity of the aluminium microstructure [2]. There were normally distributed quasi-spherical grains in the plane perpendicular to the axis of the billet, and strongly elongated grains in the plane parallel to the axis of the billet. This microstructure was due to the manufacturing process of the billets.

\section{EOS, models of fracture and recollection}

Numerical simulation of the experiments mentioned above was performed with DRAKON code [6] by using numerical schemes, which revealed the dimensions of the liner, the targets, and the momentum traps in 1D or $2 \mathrm{D}$ approximation. The measured currents $I(t)$ were used as the boundary condition to drive the liner (not taking into account possible ohmic heating of the liner).

The Mie-Grüneisen equation of state with a variable Grüneisen coefficient has been used to describe the spherical part of the stress tensor [7] of the aluminum. The deviatoric stress tensor was determined by a phenomenological relaxation model of shear strength similar to [8] with stationary yield strength being a function of two variables, cumulative plastic strain, and temperature. The melting temperature was defined according to the Lindeman's law.

The kinetic model of NAG type [9] was used to simulate the spall fracture. This model considers the fracture process as nucleation and growth of damage in the form of microdefects (micropores of spherical form), which are distributed according to a statistical law. The measure of the damage $\omega=V_{d} /\left(V_{d}+V_{s}\right)$ (where $V_{d}$-is the volume of defects, $V_{s}$ - is the volume of the solid component) is the cumulative volume of the microdefects. To determine the parameters of this model (see Table 1), we used the results of the preceding $R$ Damage experiments [2] due to the lack of data for the aluminum under study. The conception of critical damage $\omega_{\mathrm{cr}}$ was used to describe the stage of the defects' coalescence and full fracture (pressure and shear strength becomes zero in the cell) with $\omega_{c r}=0.07$. The damaged matter was considered as a two-component medium, consisted of a solid and pores. The nucleation and growth of the last were described by the kinetic equations from [9].

Table 1. Parameters of the kinetic model of spall for aluminum 1060.

\begin{tabular}{|c|c|c|c|}
\hline$P_{n 0}, \mathrm{GPa}$ & 0.57 & $\dot{N}_{0}, \mathrm{~cm}^{-3} \cdot \mathrm{s}^{-1}$ & $0.3 \cdot 10^{10}$ \\
\hline$P_{g 0}, \mathrm{GPa}$ & 0.27 & $\eta, \mathrm{Pa} \cdot \mathrm{s}$ & 60.0 \\
\hline$P_{1}, \mathrm{GPa}$ & 0.04 & $R_{0}, \mathrm{~cm}$ & $1.0 \cdot 10^{-4}$ \\
\hline
\end{tabular}

It should be mentioned that now there is no complete theory and model of the dynamic recollection of a damaged area in a medium, which was fractured by the pulse tensile stresses $[10,11]$. The development of such theory is limited, firstly, by the shortage of the experimental data needed to formulate the basic regularities of this process.

Taking this into account, two experiments of the $R$-Damage series, described above, provided the unique 
opportunity to analyse the sequence of processes, which are high-strain-rate deformation, spall fracture and recollection, by simulating numerically the targets' inner surface velocity histories $W(t)$ recorded with the help of PDV method. This simulating together with the results of post-short metallographic analysis allowed proposing the relation to close the algorithm describing the dynamics of the damaged matter.

The basic relationships of the algorithm, connected with the stages specified, are formulated as follows (see, also, Fig.4, where the stages are marked at the calculated curves):

Stage $0-1$ : nucleation and growth of damage

$$
\begin{aligned}
& \text { if }\left(\omega<\omega_{c r}\right) \cdot\left(\left(P \leq P_{n 0}\right)+\left(P \leq P_{g 0}\right)\right) \\
& \rightarrow \omega^{n+1}=f_{N A G}\left(P, \omega^{n}\right)
\end{aligned}
$$

Equations of spall fracture model [9] are used according to the condition (1) to simulate this stage. If the damage exceeds the critical level $\omega_{c r}$ the cell is considered to be fractured and full stress tensor becomes zero in it;

Stage $1-2$ : widening of the fractured cell

$$
\begin{aligned}
& \text { if }\left(\omega \geq \omega_{c r}\right) \cdot\left(P\left(\omega^{n}\right)<0\right) \rightarrow \omega^{n+1}=\left.g\left(P, \omega^{n}\right)\right|_{P \equiv 0}, \\
& \omega^{n+1} \geq \omega^{n}
\end{aligned}
$$

The damage grows in the fractured cell under tensile stresses; this growth is agreed with EOS, i.e. at each next step such damage is chosen as pressure equals zero in the cell;

Stage $2-3:$ start of recollection

$$
\begin{aligned}
& \text { if }\left(\omega \geq \omega_{0}\right) \cdot(P \geq 0) \rightarrow \omega^{n+1}=\left.\bar{g}\left(P, \omega^{n}\right)\right|_{P \equiv 0}, \\
& \omega^{n+1} \leq \omega^{n}
\end{aligned}
$$

Similar search for a smaller value of damage is performed while compressing the cell down to the limit damage $\omega_{0}$, when strength to compression is started, keeping the pressure equal zero.

Stage 3-4: recollection

$$
\begin{aligned}
& \text { if }\left(\omega_{0}>\omega>0\right) \&\left(P\left(\omega^{n}\right)>0\right) \rightarrow \\
& \rightarrow \omega^{n+1}=f_{\text {antispall }}\left(-P, \omega^{n}\right) \\
& \frac{d \omega}{d t}=-\omega \cdot \frac{P-P^{\prime}}{\eta^{\prime}} \cdot \Theta\left(P-P^{\prime}\right)
\end{aligned}
$$

After limit damage $\omega_{0}$ is achieved the further decreasing of the damage proceeds according to the condition (4), while the equation (5) acts as the function $f_{\text {antispall; }}$; here $P$ - is the positive (compressing) pressure, $P^{\prime}$ - is the pressure threshold for recollection, $\eta^{\prime}-$ is the parameter, determining the strength of the fractured material to the compression.

The advantage of the equation (5) proposed is the simplicity of its analytical form. In its turn, the algorithm provides the ample opportunities of simulating due to variation of three parameters. The results of numerical simulation, mentioned below, were obtained by using the following values of the proposed recollection algorithm parameters: $\omega_{0}=0.6, P^{\prime}=0.1 \mathrm{GPa}, \eta^{\prime}=200 \mathrm{~Pa} \cdot \mathrm{s}$.

At the same time, the equation (5) has some disadvantages. It does not take into account the elasticplastic character of the damage matter deformation process, and demands some verification when simulate the recollection process under quasi-static loading.

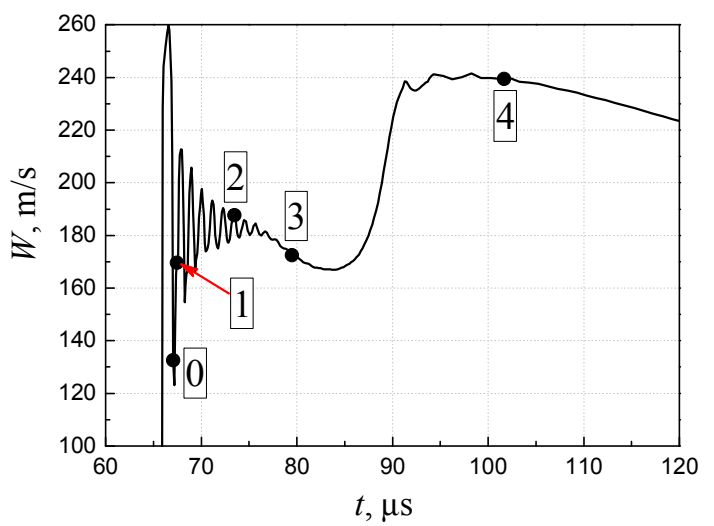

a) free surface velocity of the target vs time;

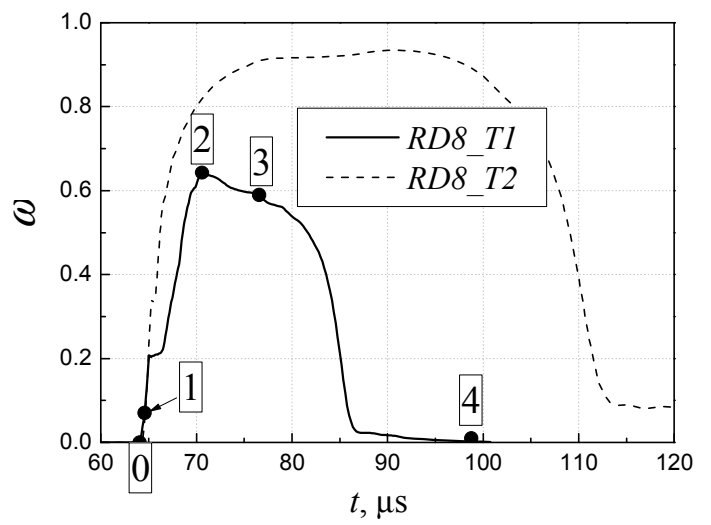

b) damage in the main crack plane vs time.

Fig. 4. Numerical simulation results for $R D 8_{-} T 1$ target.

\section{Numerical simulation}

The repeated increasing of the velocity $W$ was registered at the inner free surface velocity histories $W(t)$ for three targets - RD8_T1, RD8_T2, RD9_T1 - (see Figs. 5 \& 6) (after the first maximum, pullback and further "ringing" of spall layer). This increasing means the reacceleration of the inner surface when second compression wave arrives the surface after passing through the damage zone and recollecting it. Contrariwise, the $T 2$ target of the $R D 9$ test demonstrates a weak increasing of the velocity $W$ (time moment $\sim 120 \mu \mathrm{s}$, Fig. 6a), which is conditioned by a significant decaying of the weak recollection wave in the area of the high-level damage. It allows supposing that there is no full healing of the defects in this target. 


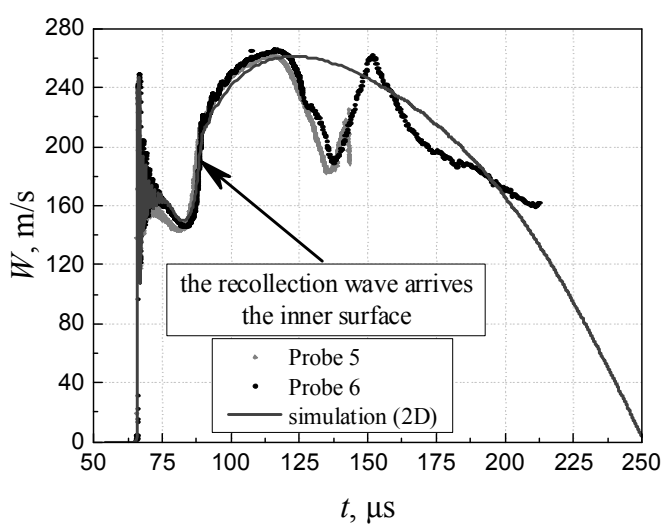

a) $R D 8 \_T 1$ target;

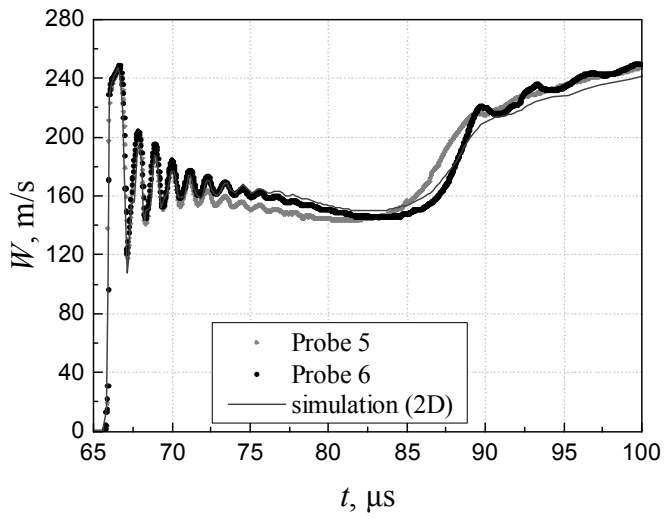

b) $R D 8 \_T 1$ target (enlarged);

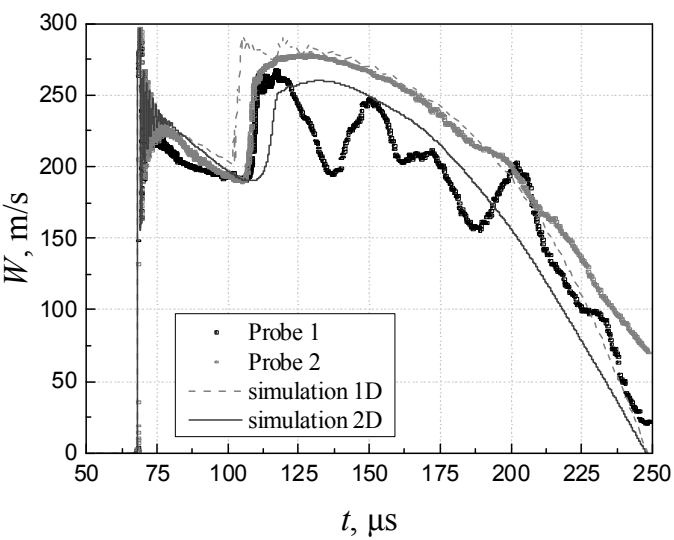

c) $R D 8 \_T 2$ target.

Fig. 5. Free surface velocity of the targets vs time.

By comparing these experimental curves with calculated ones one can see, that there is a quite adequate simulation of all the processes, occurred in the targets, if using the described above models of deformation, spall fracture and recollection. Good quantitative agreement is observed between simulation and experiments both of first maximum and of first minimum of the velocity $W(t)$, which are connected with the damage' nucleation and growth, as well as further oscillation of the spall layer (Fig.5b). Subsequent deceleration of the spall layer during axi-symmetric convergence is well simulated too. In its turn, the proposed equation of the damage decreasing (5) allows simulating the dynamics of the compression wave passing the damage zone and the "isentropic" character of the reacceleration of the inner surface while this wave arrives it (see Figs. $5 \& 6$ ). The agreement of the calculated and experimental curves in case of $T 2$ target of $R D-9$ test is well enough too. According to the calculation, the recollection wave intensity is sufficient to a short (approx. $20 \mu \mathrm{s}$ ) deceleration of the converging process only, not to a weak reacceleration of the inner surface as the experimental curve demonstrates.

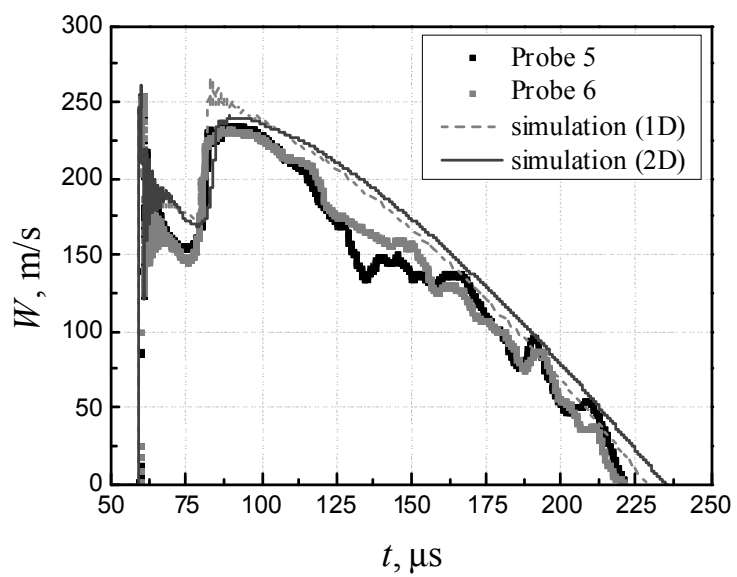

a) $R D 9 \_T 1$ target;

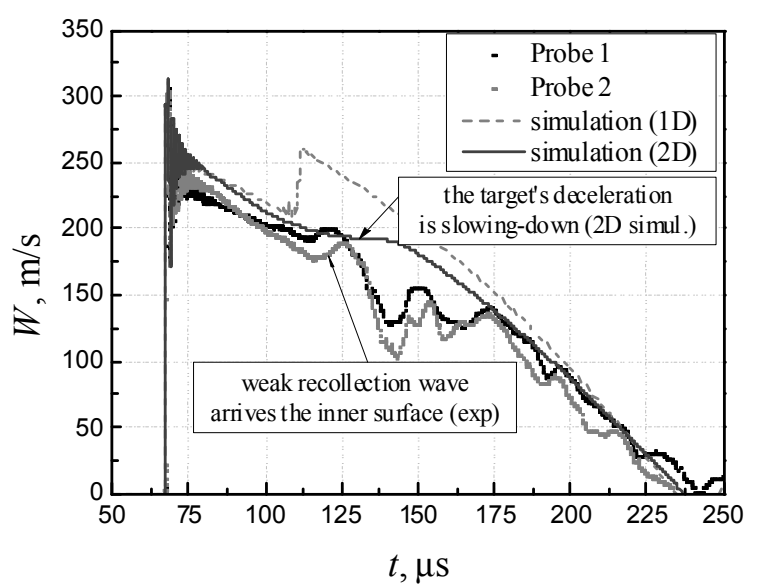

b) RD9_T2 target.

Fig. 6. Free surface velocity of the targets vs time.

The motion of the targets after $\sim 130 \mu \mathrm{s}$ is significantly non-one-dimensional one, as can be seen in the shape of the recovered targets, revealed after they were cut (see Fig. 3), due to the considerable extent of convergence. That's why the experimental curves $W(t)$ after this time are in better agreement with the results of $2 \mathrm{D}$ simulation, where axial movement is taking into account.

2D simulation described the post-shot shape of the recovered targets also. If comparing the photo of the cross-sections of the RD9_T2 target and calculated distribution of damage at Fig. 7, the position of the area of non-recollected damage is well fit with experiments.

The principal difference of the recollection process of four targets under consideration is illustrated in Fig. $4 \mathrm{~b}$ $\& 8$ by calculated dependencies of the damage vs time $\omega(t)$ in the plane of main crack. One can see the histories of growing and recollecting of damage which are similar enough for the targets of $R D-8$ test (see Fig. $4 \mathrm{~b}$ ). But for the targets of $R D-9$ test the $\omega(t)$ dependencies are rather 
different when damage decreases (Fig.8). In RD9_T2 target the amplitude of the recollection wave, which is determined by the re-impacting velocity of the liner, is not enough for full recollection of the main crack. Thus only partial recollection of the damage is realized in this target.

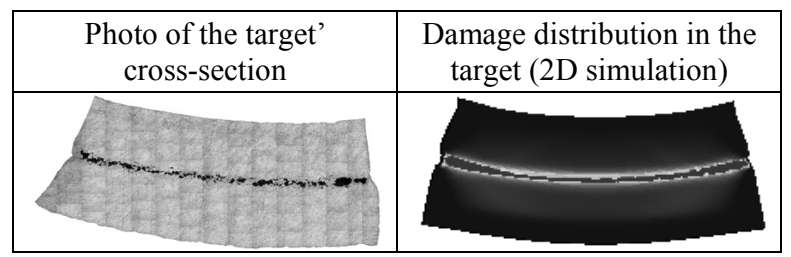

Fig. 7. Post-shot state of the target RD9 T2 vs simulation (inner surface is bottom).

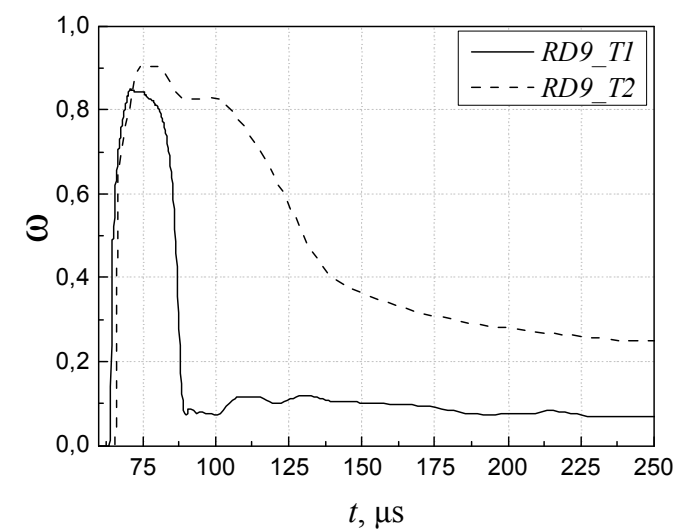

Fig. 8. Calculated histories of damage $\omega$ in the main crack plane vs time.

Figure 9 illustrates the simulated radius-time diagrams for RD9 T2 targets, including trajectories $R(t)$ of the outer and inner surfaces (solid lines) of the target and the boundaries of the damage zone (dashed lines) inside the target. Good agreement between calculated final thickness of the target and the post-short measurements of the targets' outer and inner radii (marked by stars) is observed.

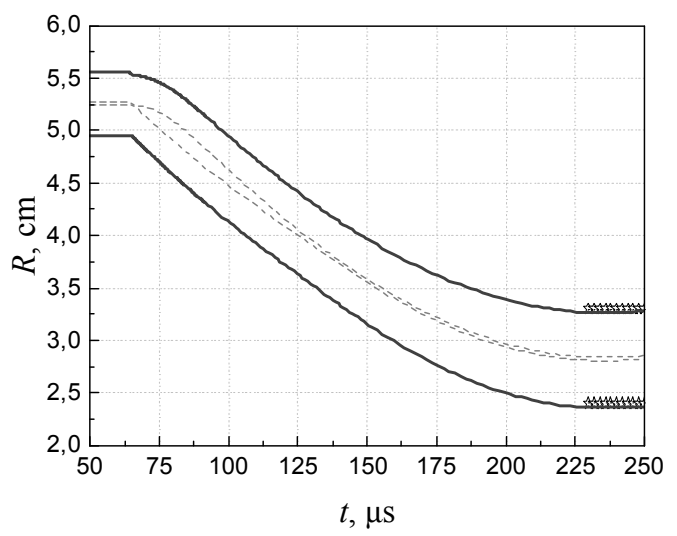

Fig. 9. $R$ - $t$ diagram for the target $R D 9+T 2$ (2D simulation results, solid lines are the outer and inner surfaces of the target, dashed lines are the boundaries of the damaged zone inside the targets).

\section{Conclusion}

Two final experiments of the R-Damage series devoted to studying the peculiarities of the damage growth and recollection under axisymmetric convergence provided a unique volume of experimental data. The results of the post-shot metallographic analysis together with PDV data allows asserting that the damage recollection of different extent - from the main crack collapsing up to partial decreasing of the damage zone size (RD9_T2) was realized in the targets.

Basing on the numerical simulation of the experimental data the algorithm to calculate the damage matter's dynamics was proposed. The whole scope of the results of dynamic measurements (the targets' inner surface velocity histories) and post-shot metallographic study was simulated quite well. This testifies to the correctness of the mathematical models used. In particular, the range of consecutive processes - highstrain rate deformation, spall fracture, deceleration of the spall layer due to axi-symmetric convergence and recollection of the damaged matter while recompression - was successfully simulated. Different levels of recollection, captured in two cylindrical targets of one test, allowed revealing the influence of the damage onto the compression behaviour of the damaged material at the finishing stage of the recollection process. It also made possible to choose the analytical dependence describing this stage of the recollection process and to define the parameters of this dependence for the material under study.

The authors are very thanks to the LANL and VNIIEF teams whose common efforts made the R-Damage series successful. Special thanks to our colleagues A.M.Podurets and A.N.Balandina for the results of metallographic analysis.

\section{References}

1. P.V. Duday, A.V. Ivanovsky, O.A. Tyupanova et al, XIII Khariton's Topical Readings, 236 (2011)

2. A.M. Kaul, A.V. Ivanovsky, W.L. Atchison et al, J. Appl.Phys. 115, 023516 (2014)

3. P.V. Duday, V.A. Ivanov, A.V. Ivanovsky et al, VIII Khariton's Topical Readings. 503 (2006)

4. P.V. Duday, A.V. Ivanovsky, O.A. Tyupanova et al, Megagauss-XIII, ISBN978-7-81099-999, 87 (2010)

5. O.T. Strand, D.R. Goosman, C. Martinez et al, Rev. Sci. Instrum. 77, 083108 (2006)

6. A.I. Abakumov, P.N. Nizovtsev, A.V. Pevnitsky, et al, IV Zababakhin's Readings, 227 (1995)

7. L.V. Altshuler, S.E. Brusnikin, High Temp. 27, 39 (1989)

8. A.P. Bolshakov, B.L. Glushak, O.N. Ignatova et al, VII Khariton's Topical Readings, 421 (2005)

9. L. Seaman, D. Curran, A. Shockey, J.Appl.Phys. 47, 4814 (1976)

10. S.S. Sokolov, VANT «Mathematical mofeling of physical processes». 4, 13 (2012)

11. V.A. Raevsky, O.N. Ignatova, I.S. Tselikov, $X V$ Khariton's Topical Readings, 498 (2013). 
